Sallbe, Gel, Schaum

\section{Topische Psoriasis-Therapie wird angenehmer}

Keine Veranstaltung und keine Studienplanung ohne Einbezug der Patienten - Leo Pharma setzt auf Anwenderorientierung, betonte Geschäftsführer Dr. Franz-Peter Kesseler. Er verwies auf Serviceangebote wie die Plattform für Psoriasis-Patienten (www.qualitycareschuppenflechte.de), aber auch auf die klinischen Entwicklungen.

Ein Beispiel ist die topische Therapie der leichten bis mittelschweren PlaquePsoriasis mit dem Kombipräparat aus Calcipotriol und Betamethason (Daivobet $^{\circledast}$ ). Patienten schilderten die Anwendung von Salben als lästig und zeitraubend, weshalb Leo ein anwenderfreundliches Gel entwickelte. Zusammen mit

\title{
Adipöse Nicht-Diabetiker
}

\section{GLP-1-Agonist unterstützt Gewichtsabnahme}

_ Das GLP-1-Analogon Liraglutid wird bereits erfolgreich in der Therapie des Typ-2-Diabetes eingesetzt. Den gewichtsreduzierenden Effekt, der vor allem über eine Appetitminderung erklärt wird, will man laut Prof. Andreas Hamann, Bad Homburg, jetzt auch bei adipösen Nicht-Diabetikern nutzen.

Im Rahmen der SCALE-Study Obesity and Prediabetes [N Engl J Med. 2015;371:11-22] wurden 3.731 Erwachsene mit einem BMI $\geq 30 \mathrm{~kg} / \mathrm{m}^{2}$ (oder $\geq 27$ mit zusätzlichen Risikofaktoren wie Dyslipidämie oder Hypertonie) mit langsam auf $3 \mathrm{mg} / \mathrm{d}$ auftitrierten Liraglutid-Injektionen oder Placebo behandelt. Zusätzlich wurde eine kalorienreduzierte Ernährung mit einem Minus von $500 \mathrm{kcal}$ und vermehrte körperlicher Bewegung empfohlen. Alle Teilnehmer hatten bereits gescheiterte Diäten hinter sich.

Nach 56 Wochen hatten die Teilnehmer der Liraglutid-Gruppe im Schnitt 8\% ihres Körpergewichts verloren, in der Placebogruppe waren es dagegen nur 2,6\%. Die Rate an

.

LAMA/LABA-Kombi bringt COPD-Patienten in Bewegung $\rightarrow$ Die Fixkombination aus dem lang wirksamen $\beta 2$-Adrenozeptor-Agonisten (LABA) Indacaterol und dem lang wirksamen Muskarinrezeptorantagonisten (LAMA) Glycopyrronium reduziert die Überblähung der Lunge und fördert die körperliche Aktivität von Patienten mit chronisch obstruktiver Lungenerkrankung (COPD). Das ist das Ergebnis der MOVE-Studie mit 191 Patienten mit moderater bis schwerer COPD. Sie erhielten nach einer Wash-out-Phase entweder die Fixkombi aus Indacaterol/Glycopyrronium (Ultibro ${ }^{\oplus}$ Breezhaler ${ }^{\circledast}$ ) oder Placebo. Die körperliche Aktivität wurde mit einem speziellen Armband gemessen. Es gab keine Lebensstil-Unterweisungen.

Nach 21 Tagen lag die Inspirationskapazität in der Verumgruppe im Mittel $202 \mathrm{ml}$ über jener der Placebogruppe. Die Verum-Patienten waren zudem körperlich aktiver: Ihr täglicher Kalorienverbrauch lag um 37 kcal höher. Die Fixkombi habe das Potenzial, auch ohne Lebensstilintervention die Überblähung zu reduzieren und die körperliche Aktivität zu steigern.

Dr. Michael Hubert

- Watz et al.: Effects of indacaterol/glycopyrronium on lung function and physical activity in patients with moderate to severe COPD. Poster beim Wintermeeting der British Thoracic Society; London, 2.-4. Dezember 2015

Per Smartphone-App mit dem Diabetes-Team vernetzt $\rightarrow$ Das Blutzuckermesssystem OneTouch Verio Flex ${ }^{\mathrm{TM}}$ wird seit Neuestem durch die OneTouch ${ }^{\circledast}$ Reveal $^{\circledR}$ Mobile App ergänzt, die den Messwert drahtlos auf das Smartphone überträgt. Anhand der mit dem Messgerät übereinstimmenden farbkodierten Bereichsanzeige kann der Patient sofort erkennen, welche Werte absolut und relativ in welchen Bereich gefallen sind. Eine Tagebuchfunktion ordnet alle Werte nach Datum und Tageszeit an. Eine Reminder-Funktion kann den Nutzer an die Blutzuckertests, die Einnahme von Mahlzeiten oder den Kauf neuer Teststreifen erinnern.

Zudem kann der Patient mit der Mobile App entscheiden, seine Messwerte an den Arzt und die Diabetesberaterin zu übertragen.

Abdol A. Ameri

- Media-Talk "Teamarbeit - Therapiemanagement des Diabetes mellitus aus verschiedenen Perspektiven", Diabetes Herbsttagung; Düsseldorf, 6. November 2015 (Veranstalter: Johnson \& Johnson Diabetes Solutions) 